OPEN ACCESS

Edited by:

Chengfei Liu,

UC Davis Medical Center,

United States

Reviewed by:

Gary Steinberg,

New York University, United States

Bogdan Geavlete,

St. John Hospital Emergency Clinic,

Romania

${ }^{*}$ Correspondence:

Ying-Hui Jin

jinyinghuiebm@163.com

Xian-Tao Zeng

zengxiantao1128@163.com;

zengxiantao1128@whu.edu.cn

Specialty section: This article was submitted to

Genitourinary Oncology,

a section of the journal

Frontiers in Oncology

Received: 03 July 2021 Accepted: 07 October 2021 Published: 27 October 2021

Citation:

Wang $D-Q$, Huang $Q$, Huang $X$, Jin $Y-H$, Wang $Y-Y$, Shi $Y-X$,

Yan S-Y, Yang L, Li B-H,

Liu T-Z and Zeng X-T (2021)

Knowledge of and Compliance

With Guidelines in the Management of Non-Muscle-Invasive Bladder Cancer:

A Survey of Chinese Urologists.

Front. Oncol. 11:735704.

doi: 10.3389/fonc.2021.735704

\section{Knowledge of and Compliance With Guidelines in the Management of Non-Muscle- Invasive Bladder Cancer: A Survey of Chinese Urologists}

\author{
Dan-Qi Wang ${ }^{1,2}$, Qiao Huang ${ }^{1}$, Xing Huang ${ }^{1,2}$, Ying-Hui Jin ${ }^{1 *}$, Yun-Yun Wang ${ }^{1}$, \\ Yue-Xian Shi ${ }^{3}$, Si-Yu Yan ${ }^{1}$, Lu Yang ${ }^{4}$, Bing-Hui Li ${ }^{1,2}$, Tong-Zu Liu ${ }^{2}$ and Xian-Tao Zeng ${ }^{1,2 *}$ \\ ${ }^{1}$ Country Center for Evidence-Based and Translational Medicine, Zhongnan Hospital of Wuhan University, Wuhan, China, \\ 2 Department of Urology, Institute of Urology, Zhongnan Hospital of Wuhan University, Wuhan, China, ${ }^{3}$ School of Nursing, \\ Peking University, Beijing, China, ${ }^{4}$ Department of Urology, Institute of Urology, West China Hospital of Sichuan University, \\ Chengdu, China
}

Background: Non-muscle-invasive bladder cancer (NMIBC) still poses a heavy load for resulting in many new cases which contribute significantly to medical costs. Although many NMIBC guidelines have been developed, their implementation remains deficient.

Objective: This study was conducted in order to analyze the knowledge of and compliance with the guidelines for NMIBC of Chinese urologists and to identify associated factors.

Methods: We conducted an online survey between August 2019 and January 2021. Respondents who were more than 65 years old or did not give informed consent were excluded. Linear/logistic regressions were performed to identify factors associated with the knowledge of and compliance with the guidelines of urologists, respectively. McNemar's tests were used to explore the divergence between knowledge and compliance.

Results: A total of 814 responses were received, and $98.77 \%$ of urologists acknowledged the positive effects of high-quality guidelines. The average knowledge score was $6.10 \pm 1.28$ (out of a full score of 9), and it was positively associated with educational level and the number of guidelines consulted. Only $1.61 \%$ and $39.36 \%$ of the respondents realized that the guidelines did not recommend further chemotherapy or BCG infusion for low-risk patients. There were 38.87\% and 51.84\% respondents "often" or more frequently utilizing BCG therapy for intermediate- and high-risk NMIBC patients, respectively. Divergence between knowledge and compliance in performing a second TURBT after incomplete initial resection reached statistical significance $(p<0.001)$. 
Conclusions: Although the vast majority of urologists acknowledged the positive effects of guidelines, knowledge of and compliance with some recommendations of NMIBC guidelines are still inadequate. Factors associated with guidelines, individual professionals, patients, organizations, and the environment jointly contributed to the non-compliance.

Keywords: guideline, guideline adherence, urinary bladder neoplasms, surveys and questionnaires, professional practice

\section{INTRODUCTION}

Ranking as the 10th most common cancer worldwide, bladder cancer caused an estimated 549,000 new cases and 200,000 deaths worldwide in 2018 (1). For therapeutic purposes, papillary tumors confined to the mucosa (Ta) or submucosa (T1) and carcinoma in situ (CIS, Tis) are classified as nonmuscle-invasive bladder cancer (NMIBC) (2), which accounts for approximately $75 \%$ of bladder cancer cases (3). NMIBC still poses a heavy load due to its high 5-year recurrence (up to 78\%) and progression (up to $45 \%$ ) risk (4), hence incurring high medical costs.

For the universal coverage of high-quality medical services, institutions and academies worldwide have invested substantial resources in developing treatment guidelines. The European Association of Urology (EAU), National Comprehensive Cancer Network (NCCN), and American Urological Association (AUA) have published guidelines relating to NMIBC, and all of them updated their guidelines last year. However, although the positive effects of guidelines have been demonstrated $(5,6)$, many studies have reported that guidelineprescribed care was not universally introduced into clinical management of $\operatorname{NMIBC}(5,7,8)$, especially the use of intravesical adjuvant therapy $(8-10)$.

The knowledge, attitude, and behavior of urologists have a great impact on the process from guideline to practice (11). However, currently, there are insufficient studies on variations in quality of bladder cancer care, and limited attention has been paid to barriers that urologists meet with in clinical practice. In this study, we collected responses of urologists with the following aims: i) to identify the knowledge and compliance rate with NMIBC guidelines; ii) to reveal the variation between knowledge and compliance, guideline, and clinical practice; and iii) to find reasons and explanations for non-compliance. To remove the impact of preference of the patient, the compliance with the guidelines of a urologist was defined as the extent to which his clinical suggestion coincided with guideline recommendations (12). It was noted that guidelines did not have any legislative influence or override the responsibility to make decisions.

\section{METHODS}

\section{Study Design}

Applying convenience and snowball sampling, we conducted an online survey on the knowledge of and compliance with NMIBC guidelines of Chinese urologists from October 2019 to January
2021. The questionnaire link was sent via a QR code posted on several national urological specialist forums in China. Meanwhile, we requested respondents to help us disseminate the questionnaire. Information for informed consent included the statement that individual responses would remain anonymous. Respondents who were more than 65 years old or did not give informed consent were excluded. No incentive was offered for participation.

\section{Development of the Questionnaire}

An exhaustive online literature review of NMIBC guidelines worldwide was conducted. We had appraised the quality of NMIBC guidelines within the past 5 years and compared the similarities and differences of therapeutic recommendations between guidelines previously (13). Based on this, six core interventions pertaining to the management of NMIBC were formulated, which addressed the initial transurethral resection of bladder tumor (TURBT), second resection, immediate postoperative intravesical instillation of chemotherapy, additional adjuvant intravesical chemotherapy instillations, intravesical bacillus Calmette-Guerin (BCG) immunotherapy, and radical cystectomy. After several rounds of group meetings, the draft was sent to three clinical urological experts and two methodologists for consultation to verify the face validity. Based on the valuable opinions of experts, we deleted the item of "device-assisted chemotherapy" and added the interpretation for "BCG failure." Through these efforts, we enhanced the acceptability and clarity of the questionnaire and also improved its expression to promote understanding.

Finally, the questionnaire consisted of the following six sections: i) demographic characteristics of urologists; ii) attitude toward guidelines in general, where the information of preferred guideline developers were gathered; iii) clinical utilization of chemotherapy and BCG; iv) knowledge of guidelines, in which the knowledge score of recommendations in NMIBC guidelines was assessed by nine items, while each item scored 1 point. Regarding the indications for surgery, given that all of them were extracted from the guidelines, 1 score was divided equally among each option (for example, if there were five indications for a second TURBT, then 0.2 point was given for each indication selected); v) compliance with guidelines, which focused on the strategy of chemotherapy and BCG for patients of each risk classification (low risk, intermediate risk, and high risk); and vi) barriers in clinical practice, which involved the barriers that respondents met in the clinical practice of a second TURBT, BCG, and radical cystectomy (Appendix 1). Urologists were asked to use yes/no/unsure or check choices of medical 
interventions to indicate whether they thought a medical intervention was recommended by guidelines or whether they would comply with it. Questions about barriers to guideline implementation were multiple choice. When asking about frequency, Likert-scale questions were adopted on a 5-point scale as never, seldom, sometimes, often, and always. Text forms were offered where extra information was needed.

\section{Statistical Analysis}

Descriptive statistics were used to summarize the data, where categorical variables were expressed as counts and percentages, and continuous variables were expressed as mean and standard deviation. McNemar's tests were used to compare the knowledge and compliance in each item. To identify characteristic factors associated with knowledge, we performed univariable and multivariable linear regressions. While univariable and multivariable logistic regressions were conducted for factors associated with compliance, Firth's logistic regressions were applied for unbalanced event with potential complete or quasicomplete separation issue. Statistical analyses were performed using SAS software, version 9.4 TS1M6 (SAS Institute Inc., Cary, NC), and a two-sided $p$-value of $\leq 0.05$ was considered statistically significant.

\section{RESULTS}

\section{Characteristics of the Respondents}

A total of 814 responses were received, 2 of which were excluded for overage and informed disconsent, respectively. The vast majority of respondents were working in tertiary hospitals $(78.45 \%)$, had been in practice for more than 15 years (63.18\%), and had a master's degree or above $(56.28 \%)$ (Table 1). Although only $10.71 \%$ respondents reported having had prior experience in guideline development, most respondents (98.77\%) agreed that high-quality guidelines were efficacious in improving healthcare quality, standardizing the clinical procedures and quality assurance. Respondents preferred guidelines developed by the Chinese Medical Association (CMA) (72.78\%), European Association of Urology (EAU) (60.96\%), and American Urological Association (AUA) (48.77\%). Respondents usually referred to an average of $2.78( \pm 1.46)$ NMIBC guidelines.

\section{Clinical Utilization of Chemotherapy and BCG}

There were $79.25 \%$ of the respondents who reported that lowrisk NMIBC patients agreed to have chemotherapy for immediate course together with maintenance course. Respondents who reported that they "often" or more frequently utilized BCG therapy for intermediate- and highrisk NMIBC patients were only $38.87 \%$ and $51.84 \%$, respectively.

For patients from each risk classification, at least $49.32 \%$ respondents scheduled induction course of intravesical chemotherapy instillations of 4 to 8 weeks, and $47.62 \%$ or more respondents scheduled a maintenance course of 6 to 12
TABLE 1 | Characteristics of the respondents.

\begin{tabular}{|c|c|c|}
\hline & $n$ & $\%$ \\
\hline \multicolumn{3}{|l|}{ Gender } \\
\hline Male & 796 & $98.03 \%$ \\
\hline Female & 16 & $1.97 \%$ \\
\hline \multicolumn{3}{|l|}{ Age } \\
\hline$<40$ & 285 & $35.10 \%$ \\
\hline $40-59$ & 515 & $63.42 \%$ \\
\hline$\geq 60$ & 12 & $1.48 \%$ \\
\hline \multicolumn{3}{|l|}{ Years of clinical practice } \\
\hline$<15$ & 299 & $36.82 \%$ \\
\hline $15-29$ & 404 & $49.75 \%$ \\
\hline$\geq 30$ & 109 & $13.42 \%$ \\
\hline \multicolumn{3}{|l|}{ Level of healthcare institute } \\
\hline 3 & 637 & $78.45 \%$ \\
\hline 2 & 174 & $21.43 \%$ \\
\hline 1 & 3 & $0.37 \%$ \\
\hline \multicolumn{3}{|l|}{ Education background } \\
\hline Doctor & 195 & $24.01 \%$ \\
\hline Master & 262 & $32.27 \%$ \\
\hline Bachelor & 345 & $42.49 \%$ \\
\hline College & 10 & $1.23 \%$ \\
\hline \multicolumn{3}{|l|}{ Professional title } \\
\hline Senior title & 233 & $28.69 \%$ \\
\hline Vice-senior title & 299 & $36.82 \%$ \\
\hline Middle title & 119 & $14.66 \%$ \\
\hline Primary title & 61 & $7.51 \%$ \\
\hline \multicolumn{3}{|l|}{ Preferred guideline developer } \\
\hline Chinese Medical Association (CMA) & 591 & $72.78 \%$ \\
\hline European Association of Urology (EAU) & 495 & $60.96 \%$ \\
\hline American Urological Association (AUA) & 396 & $48.77 \%$ \\
\hline National Comprehensive Cancer Network (NCCN) & 298 & $36.70 \%$ \\
\hline Canadian Urological Association (CUA) & 267 & $32.88 \%$ \\
\hline Chinese Medical Doctor Association (CMDA) & 236 & $29.06 \%$ \\
\hline National Institute for Health and Care Excellence (NICE) & 86 & $10.59 \%$ \\
\hline Chinese Research Hospital Association (CRHA) & 51 & $6.28 \%$ \\
\hline Have participated in guideline development & 87 & $10.71 \%$ \\
\hline Recognized the positive function of guidelines & 802 & $98.77 \%$ \\
\hline
\end{tabular}

months (Appendix 2). As for BCG, many respondents (from $42.80 \%$ to $48.14 \%$ ) scheduled induction course of BCG instillations of 6 to 8 weeks, while a maintenance course of 4 weeks or more was chosen by at least $61.98 \%$ respondents. The median length of maintenance installations was 1 year. Moreover, a majority of respondents (from $76.39 \%$ to $90.21 \%$ ) approved of a standard dose of BCG.

\section{Knowledge of Guideline Recommendations}

The average knowledge score of respondents was $6.10 \pm 1.28$ (out of a full score of 9). "The range of initial TURBT" (97.40\%) and "whether to perform an immediate postoperative instillation" (96.77\%) got the highest correct rate (Table 2). However, for low-risk patients, only $3.97 \%$ respondents realized that further chemotherapy was not recommended, and $43.08 \%$ respondents recognized that BCG immunotherapy was also not recommended.

\section{Compliance With Guideline Recommendations}

There were $98.39 \%$ of the respondents who tended to utilize further chemotherapy instillations for patients with low-risk 
TABLE 2 | Knowledge of and compliance with guideline recommendations.

\begin{tabular}{|c|c|c|c|c|}
\hline \multirow[t]{2}{*}{ No. } & \multirow[t]{2}{*}{ Recommendations } & \multicolumn{2}{|c|}{$N(\%)$} & \multirow[t]{2}{*}{$p$-value } \\
\hline & & Knowledge & Compliance & \\
\hline 1 & The presence of detrusor muscle in the specimen is necessary for the NMIBC patients having had initial TURBT & $786(97.40)$ & / & / \\
\hline 2 & $\begin{array}{l}\text { Perform a single postoperative instillation of intravesical chemotherapy after TURBT } \\
\text { Further chemotherapy instillations }\end{array}$ & $780(96.77)$ & / & / \\
\hline 3 & Not recommended for low-risk NMIBC patients & $32(3.97)$ & $13(1.61)$ & $<0.001$ \\
\hline 4 & Provide induction and maintenance chemotherapy instillations for intermediate-risk NMIBC patients & 735 (91.99) & $743(92.99)$ & 0.243 \\
\hline 5 & $\begin{array}{l}\text { Provide induction and maintenance chemotherapy instillations for high-risk NMIBC patients } \\
\text { BCG immunotherapy }\end{array}$ & l & $704(89.00)$ & / \\
\hline 6 & Not recommended for low-risk NMIBC patients & $336(43.08)$ & $307(39.36)$ & 0.181 \\
\hline 7 & Provide induction and maintenance chemotherapy instillations for intermediate-risk NMIBC patients & $513(66.97)$ & $518(67.62)$ & 0.685 \\
\hline 8 & Provide induction and maintenance chemotherapy instillations for high-risk NMIBC patients & $608(79.89)$ & $614(80.68)$ & 0.451 \\
\hline \multirow[t]{6}{*}{9} & Indications for a second TURBT & & & \\
\hline & After incomplete initial TURBT & $640(79.60)$ & $542(67.41)$ & $<0.001$ \\
\hline & There is no muscular layer in the first resected specimen (except for TaLG/G1 tumor and carcinoma in situ) & $598(74.38)$ & $595(74.00)$ & 0.863 \\
\hline & In T1 tumors & $347(43.16)$ & $357(44.40)$ & 0.474 \\
\hline & In G3/high-grade tumors (except for CIS) & $456(56.72)$ & $441(54.85)$ & 0.228 \\
\hline & Pathology analysis results of initial TURBT failed to determine stage or risk grading & $485(60.32)$ & $443(55.10)$ & 0.001 \\
\hline \multirow[t]{7}{*}{10} & Indications for radical cystectomy & & & \\
\hline & High-grade T1 with histological variation (micropapillary, sarcoma, small cell type) & $466(58.54)$ & $398(50.00)$ & $<0.001$ \\
\hline & $\begin{array}{l}\text { High-grade T1 with lymphatic vessel infiltration, multiple and/or large high-grade T1, high-grade T1 with bladder/prostate } \\
\text { CIS }\end{array}$ & $654(82.16)$ & $634(79.65)$ & 0.111 \\
\hline & Pathology analysis results of a second TURBT is still high-grade T1 & $491(61.68)$ & $545(68.47)$ & $<0.001$ \\
\hline & High-grade NMIBC with early recurrence within 3 months & $532(66.83)$ & $542(68.09)$ & 0.440 \\
\hline & NMIBC involving the bladder diverticulum & $331(41.58)$ & $384(48.24)$ & $<0.001$ \\
\hline & High-risk NMIBC patients with BCG failure & $590(74.12)$ & $526(66.08)$ & $<0.001$ \\
\hline
\end{tabular}

NMIBC (Table 2). Notably, the difference between the knowledge and compliance was statistically significant in items of further chemotherapy for low-risk patients $\left(p_{\text {low }}<0.001\right)$. For intermediate-risk patients, although BCG was widely recommended in the guidelines, the compliance rate $(67.62 \%)$ was much lower than that for chemotherapy (92.99\%). More respondents $(89.00 \%)$ suggested further chemotherapy for highrisk patients even though it was not supported by many guidelines.

Divergence between knowledge rate and compliance rate also existed in some items of surgery indications. In a second TURBT, the compliance rate was relatively lower than knowledge rate for the indication of "after incomplete initial TURBT" (79.60\% vs. $67.41 \%, p<0.001$ ), so did "pathology analysis results of initial TURBT failed to determine stage or risk grading" $(60.32 \%$ vs. $55.10 \%, p<0.0011)$.

\section{Characteristic Factors Associated With Knowledge and Compliance}

We used multiple linear regressions to assess characteristic factors associated with knowledge score of the respondents (Table 3). Knowledge scores were positively and independently associated with the number of guidelines that respondents usually referred to, and the adjusted coefficient was 0.17 (95\% CI, 0.10 to 0.23 ), $p<0.001$. Compared with bachelors, masters and $\mathrm{PhDs}$ were more likely to gain higher knowledge scores $(B=0.67, p<0.001 ; B=0.42, p<0.001)$. Besides, respondents from the Middle may gain lower scores than those from the East and the Northeast (we combined the data of respondents from these two regions for regression analysis) $(B=-0.28, p=0.022)$.
Univariable and multivariable logistic regressions were performed to identify factors associated with the compliance of the respondent with guideline recommendation (Tables 4,5 ). Knowledge of the recommendation was demonstrated to affect the compliance positively $(p<0.001)$. In some items, respondents who consulted more guidelines were more likely to comply with the guidelines, such as further chemotherapy and BCG therapy for patients with intermediate-risk NMIBC $(\mathrm{OR}=$ $1.32, p=0.047 ; \mathrm{OR}=1.22, p=0.015)$. However, the result of $\mathrm{BCG}$ therapy for low-risk patients was reversed $(\mathrm{OR}=0.87, p=0.034)$. Regarding further chemotherapy for intermediate-risk patients $(\mathrm{OR}=0.24, p=0.033)$ and BCG for high-risk patients $(\mathrm{OR}=$ $0.36, p=0.016$ ), respondents from the Middle were more likely to gain less score than those from the East and the Northeast.

\section{Barriers in Clinical Practice}

There were $70.63 \%$ of the respondents who reported that "patients rejected the operation because of risk or side effects" prevented the implementation of a second TURBT in clinical practice, followed by "patients rejected the operation for economic reasons" (50.43\%) and "urologist didn't suggest it because of risk or complications" (37.55\%) (Appendix 3). As data about the frequency of BCG utilization and radical cystectomy in clinical practice had been collected, we excluded respondents who "usually" or "always" implemented them in barrier analysis. For barriers that hindered the implementation of BCG, "drug was not accessible" was the dominant barrier (74.89\%). Besides, there were $62.22 \%$ respondents who thought "patients rejected the operation for economic reasons" "often", "usually", or "always" hindered the implementation. With regard 
TABLE 3 | Multivariable linear regressions of knowledge of guidelines.

\begin{tabular}{|c|c|c|c|}
\hline \multirow[t]{2}{*}{ Variables } & \multirow[t]{2}{*}{ Level } & \multicolumn{2}{|c|}{ Multivariable } \\
\hline & & Coefficient $(95 \% \mathrm{Cl})$ & $p$-value \\
\hline \multirow[t]{2}{*}{ Hospital level } & Tertiary & $0.20(-0.06,0.46)$ & 0.128 \\
\hline & Secondary and below & Reference & \\
\hline \multirow[t]{4}{*}{ Years of practice } & $11 \sim 20$ & $0.12(-0.16,0.39)$ & 0.401 \\
\hline & $21 \sim 30$ & $-0.05(-0.40,0.30)$ & 0.786 \\
\hline & $\geq 31$ & $-0.14(-0.60,0.32)$ & 0.551 \\
\hline & $\leq 10$ & Reference & \\
\hline \multirow[t]{3}{*}{ Education background } & $\mathrm{PhD}$ & $0.67(0.39,0.95)$ & $<0.001$ \\
\hline & Master & $0.67(0.39,0.95)$ & $<0.001$ \\
\hline & Bachelor or college & Reference & \\
\hline \multirow[t]{3}{*}{ Professional title } & Senior & $0.30(-0.05,0.66)$ & 0.094 \\
\hline & Vice-senior & $0.12(-0.15,0.38)$ & 0.391 \\
\hline & Middle and below & Reference & \\
\hline \multirow[t]{2}{*}{ Having participated in guideline development } & Yes & $-0.00(-0.30,0.29)$ & 0.973 \\
\hline & No & Reference & \\
\hline \multirow[t]{3}{*}{ Region } & The Middle & $-0.28(-0.51,-0.04)$ & 0.022 \\
\hline & The West & $-0.10(-0.33,0.14)$ & 0.431 \\
\hline & The East and the Northeast & Reference & \\
\hline Number of guidelines consulted & & $0.23(0.07,0.39)$ & 0.005 \\
\hline
\end{tabular}

Cl, confidence interval.

TABLE 4 | Multivariable logistic regression of compliance with recommendation about chemotherapy.

\begin{tabular}{|c|c|c|c|c|c|c|c|}
\hline \multirow[t]{3}{*}{ Independent variables } & \multirow[t]{3}{*}{ Level } & \multicolumn{6}{|c|}{ Dependent variables } \\
\hline & & \multicolumn{2}{|c|}{ Low-risk patients } & \multicolumn{2}{|c|}{ Intermediate-risk patients } & \multicolumn{2}{|c|}{ High-risk patients } \\
\hline & & OR $(95 \% \mathrm{Cl})$ & $p$-value & OR $(95 \% \mathrm{Cl})$ & $p$-value & OR $(95 \% \mathrm{Cl})$ & $p$-value \\
\hline \multirow[t]{2}{*}{ Hospital level } & Tertiary & $1.93(0.08,45.97)$ & 0.684 & $0.38(0.13,1.11)$ & 0.077 & $1.80(0.63,5.15)$ & 0.272 \\
\hline & Secondary and below & Reference & & & & & \\
\hline \multirow[t]{4}{*}{ Years of practice } & $11 \sim 20$ & $0.24(0.03,2.21)$ & 0.207 & $1.77(0.54,5.86)$ & 0.348 & $0.46(0.16,1.33)$ & 0.15 \\
\hline & $21 \sim 30$ & $0.22(0.02,3.20)$ & 0.271 & $0.87(0.18,4.23)$ & 0.86 & $1.24(0.29,5.38)$ & 0.773 \\
\hline & $\geq 31$ & $0.20(0.01,4.52)$ & 0.31 & $0.51(0.07,3.58)$ & 0.496 & $1.07(0.18,6.51)$ & 0.942 \\
\hline & $\leq 10$ & Reference & & & & & \\
\hline \multirow[t]{3}{*}{ Education background } & $\mathrm{PhD}$ & $4.42(0.37,52.56)$ & 0.239 & $5.76(1.30,25.49)$ & 0.021 & $2.48(0.67,9.16)$ & 0.174 \\
\hline & Master & $6.11(0.65,57.62)$ & 0.114 & $1.23(0.47,3.21)$ & 0.674 & $0.94(0.36,2.44)$ & 0.901 \\
\hline & Bachelor or college & Reference & & & & & \\
\hline \multirow[t]{3}{*}{ Professional title } & Senior & $3.39(0.25,46.34)$ & 0.361 & $3.25(0.61,17.31)$ & 0.167 & $2.33(0.53,10.23)$ & 0.263 \\
\hline & Vice-senior & $0.71(0.09,5.50)$ & 0.744 & $1.28(0.41,4.04)$ & 0.671 & $2.91(0.98,8.62)$ & 0.054 \\
\hline & Middle and below & Reference & & & & & \\
\hline \multirow{2}{*}{$\begin{array}{l}\text { Having participated in guideline } \\
\text { development }\end{array}$} & Yes & $0.61(0.11,3.32)$ & 0.566 & $0.15(0.05,0.47)$ & 0.001 & $0.62(0.16,2.49)$ & 0.503 \\
\hline & No & Reference & & & & & \\
\hline \multirow[t]{3}{*}{ Region } & The Middle & $1.12(0.22,5.60)$ & 0.894 & $0.24(0.07,0.89)$ & 0.033 & $1.14(0.38,3.47)$ & 0.816 \\
\hline & The West & $1.96(0.48,7.96)$ & 0.347 & $0.39(0.10,1.46)$ & 0.164 & $0.66(0.22,2.01)$ & 0.464 \\
\hline & $\begin{array}{l}\text { The East and the } \\
\text { Northeast }\end{array}$ & Reference & & & & & \\
\hline Number of guidelines consulted & & $1.12(0.75,1.68)$ & 0.573 & $1.32(1.00,1.74)$ & 0.047 & $0.79(0.60,1.05)$ & 0.099 \\
\hline Knowledge of this recommendation & & $57.71(16.77,198.60)$ & $<0.001$ & $101.59(44.85,230.12)$ & $<0.001$ & $254.97(104.95,619.46)$ & $<0.001$ \\
\hline
\end{tabular}

to radical cystectomy, $57.73 \%$ respondents indicated that "patients rejected it because of decrease in life quality" often or more frequently hindered the implementation.

Besides, studies of similar themes or about determinants of practice were also reviewed to analyze the reason for noncompliance. After reviewing studies about compliance with the guidelines, determinants of practice, and others, we developed a flowchart to incorporate the sequence of compliance and corresponding influencing factors (Figure 1) $(11,14,15)$.

\section{DISCUSSION}

Our findings demonstrated the discrepancy between guideline and practice, inadequate knowledge of guidelines, and deficient compliance with guidelines, all of which were also reported by previous studies (8-10). In the analysis of findings, we found that factors associated with guidelines, individual professionals, patients, organizations, and the environment all contributed to the transition from guideline to practice $(11,14)$. 
TABLE 5 | Multivariable logistic regression of compliance with recommendation about BCG immunotherapy.

\begin{tabular}{|c|c|c|c|c|c|c|c|}
\hline \multirow[t]{3}{*}{ Independent variables } & \multirow[t]{3}{*}{ Level } & \multicolumn{6}{|c|}{ Dependent variables } \\
\hline & & \multicolumn{2}{|c|}{ Low-risk patients } & \multicolumn{2}{|c|}{ Intermediate-risk patients } & \multicolumn{2}{|c|}{ High-risk patients } \\
\hline & & OR (95\% Cl) & $p$-value & OR (95\% Cl) & $p$-value & OR $(95 \% \mathrm{Cl})$ & $p$-value \\
\hline \multirow[t]{2}{*}{ Hospital level } & Tertiary & $0.88(0.53,1.47)$ & 0.622 & $1.54(0.81,2.95)$ & 0.19 & $2.85(1.33,6.12)$ & 0.007 \\
\hline & Secondary and below & Reference & & & & & \\
\hline \multirow[t]{4}{*}{ Years of practice } & $11 \sim 20$ & $1.51(0.87,2.60)$ & 0.14 & $1.13(0.56,2.27)$ & 0.734 & $0.77(0.33,1.82)$ & 0.559 \\
\hline & $21 \sim 30$ & $1.08(0.54,2.16)$ & 0.82 & $1.41(0.58,3.44)$ & 0.449 & $0.49(0.16,1.50)$ & 0.212 \\
\hline & $\geq 31$ & $1.06(0.42,2.67)$ & 0.907 & $2.01(0.62,6.53)$ & 0.243 & $0.50(0.12,2.05)$ & 0.333 \\
\hline & $\leq 10$ & Reference & & & & & \\
\hline \multirow[t]{3}{*}{ Education background } & PhD & $1.00(0.57,1.74)$ & 0.991 & $0.92(0.45,1.88)$ & 0.827 & $0.54(0.21,1.40)$ & 0.204 \\
\hline & Master & $1.30(0.81,2.08)$ & 0.271 & $0.91(0.50,1.65)$ & 0.76 & $0.53(0.25,1.10)$ & 0.09 \\
\hline & Bachelor or college & Reference & & & & & \\
\hline \multirow[t]{3}{*}{ Professional title } & Senior & $0.96(0.48,1.92)$ & 0.903 & $0.56(0.23,1.38)$ & 0.206 & $1.03(0.34,3.08)$ & 0.963 \\
\hline & Vice-senior & $1.15(0.69,1.93)$ & 0.593 & $0.64(0.33,1.26)$ & 0.198 & $0.91(0.41,2.03)$ & 0.813 \\
\hline & Middle and below & Reference & & & & & \\
\hline \multirow{2}{*}{$\begin{array}{l}\text { Having participated in guideline } \\
\text { development }\end{array}$} & Yes & $0.67(0.38,1.20)$ & 0.183 & $0.68(0.33,1.41)$ & 0.3 & $1.57(0.54,4.50)$ & 0.405 \\
\hline & No & Reference & & & & & \\
\hline \multirow[t]{3}{*}{ Region } & The Middle & $1.32(0.82,2.10)$ & 0.251 & $0.66(0.36,1.20)$ & 0.173 & $0.36(0.16,0.83)$ & 0.016 \\
\hline & The West & $1.35(0.84,2.17)$ & 0.209 & $0.72(0.38,1.35)$ & 0.301 & $0.70(0.29,1.65)$ & 0.409 \\
\hline & $\begin{array}{l}\text { The East and the } \\
\text { Northeast }\end{array}$ & Reference & & & & & \\
\hline Number of guidelines consulted & & $0.87(0.77,0.99)$ & 0.034 & $1.22(1.04,1.43)$ & 0.015 & $1.20(0.99,1.45)$ & 0.068 \\
\hline Knowledge of this recommendation & & $10.47(7.22,15.17)$ & $<0.001$ & $38.01(24.44,59.12)$ & $<0.001$ & $77.17(42.21,141.08)$ & $<0.001$ \\
\hline
\end{tabular}

\section{Guideline Self-Related Factors}

Guidelines need to reflect current research (16), and one out of five recommendations could be out of date after 3 years (17). CMA amended and published their guideline last October, while its previous version was published in 2014. Given that it took time for the guideline to disseminate, the CMA guideline that was familiar to respondents when filling in questionnaires might be the 2014 version. From the aspect of timeliness, it could have less credibility compared with the EAU and NCCN guidelines, which were updated every year. After all, these results show an evident preference of respondents for native guideline. A survey conducted in China explored the barriers and enablers for the implementation of guidelines, which reported that $27.3 \%$ of their respondents encountered language barriers associated with English guidelines (18). Language might be an important reason for the gap of utilization between guidelines in Chinese and non-native language. Efforts should be devoted to increase readability of guideline for non-native speakers, and to provide translated executive summaries of guidelines could be a solution. Meanwhile, it requires guideline users to build trust in nonnative guidelines and improve linguistic proficiency.

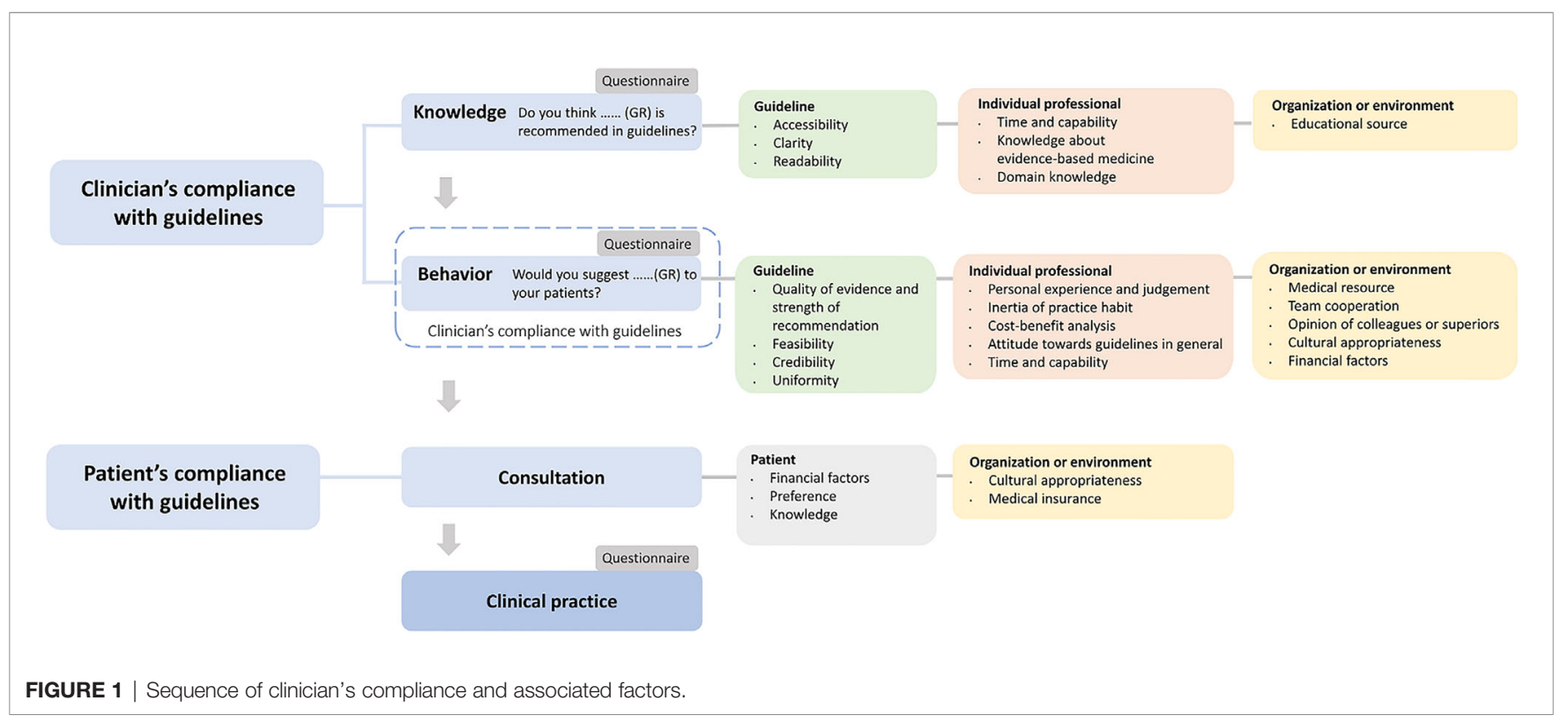


In addition, the feasibility of the guidelines is crucial for urologists to determine whether to recommend the guidelines to patients in actual clinical situations. As mentioned above, although some respondents knew that "an incomplete resection" was an indication for a second TURBT in the guidelines, they would not comply with it. Two respondents indicated that there was no objective criteria for TURBT to judge whether it was "a complete resection" or not. Hence, urologists were confused with this indication when applying guidelines. To enhance feasibility, guideline developers could give a definition to "complete resection" (for example, there was no residue with the naked eyes during operation or with cystoscopy), or detail the situations for the recommendation, such as "clear visual field in operation" and "the histopathology of the tumour base was negative".

\section{Individual Professional-Related Factors}

Knowledge of guideline is an essential prerequisite for compliance (11). For patients with low-risk NMIBC, it was stated in the guidelines that a single postoperative intravesical instillation was considered to be standard and complete treatment (19), while an additional chemotherapy course did not confer benefit but increase the risk of side effects (20). Our results indicated disturbing overuse of further intravesical instillations for low-risk patients, which reveals insufficient knowledge. Moreover, we found that respondents who referred to more guidelines were more likely to comply with guideline recommendations about the utilization of BCG for low-risk patients. Reminding urologists of the need to devote more time in acquiring guideline recommendations is essential. On the other hand, educational source about guidelines needs to be developed to benefit more urologists.

We got feedback from the respondents that the capability of communication with patients is of vital importance in clinical practice, especially when persuading patients to agree to have a second TURBT, or eschew further chemotherapy installations for low-risk NMIBC. Maintaining a harmonious relationship between patients and clinicians based on mutual trust and communication was essential (12).

Furthermore, the capability and training of urologists determined whether they could implement the recommendation in practice. In the study of Witjes et al., only $17.3 \%$ of intermediate-risk patients received BCG therapy in their group, which conformed to our results (10). Under the premise of the high toxicity and treatment cessation rate of BCG, one of the various barriers to its utilization was the lack of experience and confidence in the prevention and management of BCG-associated adverse events. As the management of BCG toxicity has been expounded in the EAU guideline (19) and practical recommendations for the prevention and management of adverse events had been acknowledged, these efforts might increase knowledge and finally contribute to clinical practice.

\section{Organization- or Environment-Related Factors}

In our study, the lack of drug accessibility was the primary barrier to the utilization of BCG. Not only some respondents reported no local supply of BCG, but also the requirements for the transportation and storage of BCG are strict, which presents a challenge to the ability of a hospital to source the medicine. Actually, BCG shortage was a global problem, especially comparing with increasing demands (21). Desouky indicated that the ongoing clinical trials using BCG against COVID-19 could aggravate the shortage and influence our urology practice (22). Under the circumstances, both the AUA and NCCN provided strategies for urologists to help mitigate the conflicts, such as using chemotherapy as a substitute in intermediate-risk NMIBC patients when BCG was second-line therapy $(20,23)$.

\section{Patient-Related Factors}

A survey conducted in Europe also reported wide overuse of additional adjuvant intravesical chemotherapy instillations for low-risk NMIBC patients (8). For patients without supporting medical knowledge, it was firmly believed that chemotherapy was an essential measure for the management of all tumors. If the tumor recurs or progresses, the urologist would be severely blamed by patients and their relatives for his "negative" attitude. Hence, medical education for patients was necessary to improve their knowledge of disease management, especially their knowledge of and confidence in the guidelines. At this point, some guidelines have included patients in their targeted users, or a specially designed patient version has been published.

Meanwhile, the financial resources of patients also influenced the implementation of recommendations. The price of BCG was much higher than chemotherapy, but it was included into the scope of reimbursement only in a few provinces of China. Our study showed that a second TURBT and radical cystectomy were also limited by the financial resource of patients. For instance, patients need to pay the cost of operation, postoperative urine collection bag, skin protection materials, and others. However, BCG therapy could achieve cost savings by decreasing the risks of local recurrence and its attendant treatments (24). A costeffectiveness study found that the implementation of BCG intravesical therapy decreased costs by $\$ 3,900$ per 5 -year recurrence-free interval compared with the total costs attributable to recurrences (25).

\section{Strengths and Limitations}

This is the first nationwide survey on the compliance of urologists with NMIBC guidelines in China, and it reveals the reasons for non-compliance in detail. The questionnaire development and distribution were carried out under the guidance of methodological experts. Through detailed analysis, it provided reliable information for guideline developers to improve the guideline implementation. However, this study still has some limitations that have to be considered. Recall bias might exist as the results were based on the subjective feedback of urologists, especially in the self-reported barriers to guideline implementation. Respondents might overstate the impact of patients and reduce their own responsibilities in non-compliance. Meanwhile, the results might be affected by the sample size of the questionnaire as well as the unknown response rate. 


\section{CONCLUSIONS}

This survey revealed that although the vast majority of urologists acknowledged the positive effects of guidelines, the knowledge of and compliance with some recommendations in NMIBC guidelines were still not high enough. It highlights the overuse of further intravesical instillations for low-risk NMIBC patients and the underuse of BCG for intermediate-risk and high-risk patients. Guideline factors (lack of readability and feasibility), individual professional factors (insufficient knowledge, lack of capability and training), organization or environment factors (lack of educational and medical resources), and patient factors (preference and financial sources) jointly resulted in the non-compliance.

\section{DATA AVAILABILITY STATEMENT}

The raw data supporting the conclusions of this article will be made available by the authors, without undue reservation.

\section{ETHICS STATEMENT}

The studies involving human participants were reviewed and approved by the Ethics Committee of Wuhan University School of Medicine. The patients/participants provided their written informed consent to participate in this study.

\section{AUTHOR CONTRIBUTIONS}

Study concept and design: Y-HJ, D-QW, and X-TZ. Acquisition of data: Y-XS, Y-YW, S-YY, LY, and B-HL. Analysis and interpretation of data: QH, D-QW, and XH. Drafting of the manuscript: D-QW,

\section{REFERENCES}

1. Bray F, Ferlay J, Soerjomataram I, Siegel RL, Torre LA, Jemal A. Global Cancer Statistics 2018: GLOBOCAN Estimates of Incidence and Mortality Worldwide for 36 Cancers in 185 Countries. CA Cancer J Clin (2018) 68 (6):394-424. doi: 10.3322/caac.21492

2. Paner GP, Stadler WM, Hansel DE, Montironi R, Lin DW, Amin MB. Updates in the Eighth Edition of the Tumor-Node-Metastasis Staging Classification for Urologic Cancers. Eur Urol (2018) 73(4):560-9. doi: 10.1016/j.eururo.2017.12.018

3. Dobruch J, Daneshmand S, Fisch M, Lotan Y, Noon AP, Resnick MJ, et al. Gender and Bladder Cancer: A Collaborative Review of Etiology, Biology, and Outcomes. Eur Urol (2016) 69(2):300-10. doi: 10.1016/j.eururo.2015.08.037

4. van Rhijn BW, Burger M, Lotan Y, Solsona E, Stief CG, Sylvester RJ, et al. Recurrence and Progression of Disease in Non-Muscle-Invasive Bladder Cancer: From Epidemiology to Treatment Strategy. Eur Urol (2009) 56 (3):430-42. doi: 10.1016/j.eururo.2009.06.028

5. Chamie K, Saigal CS, Lai J, Hanley JM, Setodji CM, Konety BR, et al. Quality of Care in Patients With Bladder Cancer: A Case Report? Cancer (2012) 118 (5):1412-21. doi: 10.1002/cncr.26402

6. Huang GJ, Hamilton AS, Lo M, Stein JP, Penson DF. Predictors of Intravesical Therapy for Nonmuscle Invasive Bladder Cancer: Results From the Surveillance, Epidemiology and End Results Program 2003 Patterns of Care Project. J Urol (2008) 180(2):520-4; discussion 4. doi: 10.1016/j.juro.2008.04.016
$\mathrm{QH}$, and Y-HJ. Critical revision of the manuscript for important intellectual content: XH, Y-HJ, and T-ZL. Obtaining funding: Y-HJ and X-TZ. Administrative, technical, or material support: LY and XTZ. Supervision: T-ZL and X-TZ. All authors contributed to the article and approved the submitted version.

\section{FUNDING}

This work was supported (in part) by the Planning Project of Innovation and Entrepreneurship Training of Undergraduate of Wuhan University (S2019303011), the National Natural Science Foundation of China (82174230), and the National Key Research and Development Program of China (Science and Technology Powers Economy 2020; 2016YFC0106300).

\section{ACKNOWLEDGMENTS}

The authors thank all those urologists across China who participated in this study and generously gave their time and shared their opinions. The authors also thank Zhi-Min Chen, who works in the Department of Social Medical Development, Zhongnan Hospital of Wuhan University. The authors express their gratitude to Jean Glover from Tianjin Golden Framework Consulting Company for English editing.

\section{SUPPLEMENTARY MATERIAL}

The Supplementary Material for this article can be found online at: https://www.frontiersin.org/articles/10.3389/fonc.2021. 735704/full\#supplementary-material

7. Chamie K, Saigal CS, Lai J, Hanley JM, Setodji CM, Konety BR, et al. Compliance With Guidelines for Patients With Bladder Cancer: Variation in the Delivery of Care. Cancer (2011) 117(23):5392-401. doi: 10.1002/cncr.26198

8. Hendricksen K, Aziz A, Bes P, Chun FK, Dobruch J, Kluth LA, et al. Discrepancy Between European Association of Urology Guidelines and Daily Practice in the Management of Non-Muscle-Invasive Bladder Cancer: Results of a European Survey. Eur Urol Focus (2019) 5(4):681-8. doi: 10.1016/ j.euf.2017.09.002

9. Gontero P, Oderda M, Altieri V, Bartoletti R, Cai T, Colombo R, et al. Are Referral Centers for Non-Muscle-Invasive Bladder Cancer Compliant to EAU Guidelines? A Report From the Vesical Antiblastic Therapy Italian Study. Urol Int (2011) 86(1):19-24. doi: 10.1159/000321926

10. Witjes JA, Palou J, Soloway M, Lamm D, Kamat AM, Brausi M, et al. Current Clinical Practice Gaps in the Treatment of Intermediate- and High-Risk NonMuscle-Invasive Bladder Cancer (NMIBC) With Emphasis on the Use of Bacillus Calmette-Guérin (BCG): Results of an International Individual Patient Data Survey (IPDS). BJU Int (2013) 112(6):742-50. doi: 10.1111/bju.12012

11. Cabana MD, Rand CS, Powe NR, Wu AW, Wilson MH, Abboud PA, et al. Why Don't Physicians Follow Clinical Practice Guidelines? A Framework for Improvement. Jama (1999) 282(15):1458-65. doi: 10.1001/jama.282.15.1458

12. Diamond-Brown L. The Doctor-Patient Relationship as a Toolkit for Uncertain Clinical Decisions. Soc Sci Med (2016) 159:108-15. doi: 10.1016/ j.socscimed.2016.05.002 
13. Zhang J, Wang Y, Weng H, Wang D, Han F, Huang Q, et al. Management of Non-Muscle-Invasive Bladder Cancer: Quality of Clinical Practice Guidelines and Variations in Recommendations. BMC Cancer (2019) 19(1):1054. doi: 10.1186/s12885-019-6304-y

14. Flottorp SA, Oxman AD, Krause J, Musila NR, Wensing M, Godycki-Cwirko M, et al. A Checklist for Identifying Determinants of Practice: A Systematic Review and Synthesis of Frameworks and Taxonomies of Factors That Prevent or Enable Improvements in Healthcare Professional Practice. Implement Sci (2013) 8:35. doi: 10.1186/1748-5908-8-35

15. Gagliardi AR, Armstrong MJ, Bernhardsson S, Fleuren M, Pardo-Hernandez H, Vernooij RWM, et al. The Clinician Guideline Determinants Questionnaire was Developed and Validated to Support Tailored Implementation Planning. J Clin Epidemiol (2019) 113:129-36. doi: 10.1016/j.jclinepi.2019.05.024

16. Dans AL, Dans LF. Appraising a Tool for Guideline Appraisal (the AGREE II Instrument). J Clin Epidemiol (2010) 63(12):1281-2. doi: 10.1016/ j.jclinepi.2010.06.005

17. Martínez García L, Sanabria AJ, García Alvarez E, Trujillo-Martín MM, Etxeandia-Ikobaltzeta I, Kotzeva A, et al. The Validity of Recommendations From Clinical Guidelines: A Survival Analysis. Cmaj (2014) 186(16):1211-9. doi: 10.1503/cmaj.140547

18. Jin Y, Li Z, Han F, Huang D, Huang Q, Cao Y, et al. Barriers and Enablers for the Implementation of Clinical Practice Guidelines in China: A MixedMethod Study. BMJ Open (2019) 9(9):e026328. doi: 10.1136/bmjopen-2018026328

19. Babjuk MB M, Compérat E, Gontero P, Mostafid AH, Palou J, van Rhijn BWG, et al. EAU Guidelines on Non-Muscle-Invasive Bladder Cancer (Tat1 and CIS). Arnhem, The Netherlands: European Association of Urology (2020). Available at: https://uroweb.org/guideline/non-muscle-invasivebladder-cancer/.

20. Chang SS, Boorjian SA, Chou R, Clark PE, Daneshmand S, Konety BR, et al. Diagnosis and Treatment of Non-Muscle Invasive Bladder Cancer: AUA/SUO Joint Guideline. Linthicum, Maryland: American Urological Association Education and Research, Inc. (2020). Available at: https://www.auanet.org/ guidelines/bladder-cancer-non-muscle-invasive-guideline\#x2516.
21. Cernuschi T, Malvolti S, Nickels E, Friede M. Bacillus Calmette-Guérin (BCG) Vaccine: A Global Assessment of Demand and Supply Balance. Vaccine (2018) 36(4):498-506. doi: 10.1016/j.vaccine.2017.12.010

22. Desouky E. BCG Versus COVID-19: Impact on Urology. World J Urol (2021) 39(3):823-7. doi: 10.1007/s00345-020-03251-7

23. Flaig TW, Spiess PE, Agarwal N, Bangs R, Boorjian SA, Buyyounouski MK, et al. Bladder Cancer, Version 3.2020, NCCN Clinical Practice Guidelines in Oncology. J Natl Compr Cancer Netw: JNCCN (2020) 18(3):329-54. doi: 10.6004/jnccn.2020.0011

24. Svatek RS, Hollenbeck BK, Holmäng S, Lee R, Kim SP, Stenzl A, et al. The Economics of Bladder Cancer: Costs and Considerations of Caring for This Disease. Eur Urol (2014) 66(2):253-62. doi: 10.1016/j.eururo.2014.01.006

25. Uchida A, Yonou $\mathrm{H}$, Hayashi E, Iha K, Oda M, Miyazato M, et al. Intravesical Instillation of Bacille Calmette-Guérin for Superficial Bladder Cancer: CostEffectiveness Analysis. Urology (2007) 69(2):275-9. doi: 10.1016/ j.urology.2006.10.017

Conflict of Interest: The authors declare that the research was conducted in the absence of any commercial or financial relationships that could be construed as a potential conflict of interest.

Publisher's Note: All claims expressed in this article are solely those of the authors and do not necessarily represent those of their affiliated organizations, or those of the publisher, the editors and the reviewers. Any product that may be evaluated in this article, or claim that may be made by its manufacturer, is not guaranteed or endorsed by the publisher.

Copyright (C) 2021 Wang, Huang, Huang, Jin, Wang, Shi, Yan, Yang, Li, Liu and Zeng. This is an open-access article distributed under the terms of the Creative Commons Attribution License (CC BY). The use, distribution or reproduction in other forums is permitted, provided the original author(s) and the copyright owner(s) are credited and that the original publication in this journal is cited, in accordance with accepted academic practice. No use, distribution or reproduction is permitted which does not comply with these terms. 